\title{
Piezospin Polarization of Currents in Nanostructures
}

\author{
Alexey A. Kovalev, ${ }^{1}$ Liviu P. Zârbo, ${ }^{1}$ Yaroslav Tserkovnyak, ${ }^{2}$ Gerrit E. W. Bauer, ${ }^{3}$ and Jairo Sinova ${ }^{1}$ \\ ${ }^{1}$ Department of Physics, Texas A\&M University, College Station, Texas 77843-4242, USA \\ ${ }^{2}$ Department of Physics and Astronomy, University of California, Los Angeles, California 90095, USA \\ ${ }^{3}$ Kavli Institute of NanoScience, Delft University of Technology, Lorentzweg 1, 2628CJ Delft, The Netherlands
}

(Received 28 November 2007; published 16 July 2008)

\begin{abstract}
Torsional oscillations of a freestanding semiconductor beam are shown to cause spin-dependent oscillating potentials that spin polarize an applied charge current in the presence of intentional or disorder scattering potentials. We propose several realizations of mechanical spin generators and manipulators based on this piezospintronic effect.
\end{abstract}

DOI: 10.1103/PhysRevLett.101.036401

Spintronics comprises the search for novel logic and sensing devices that employ the electron spin degree of freedom by (excess) spin generation and manipulation [1]. In the conventional approach, spins are injected into normal conductors by ferromagnetic metals using an applied electrical bias. Another method is the spin-pumping by a moving magnetization [2,3]. A net-spin generation is possible without involving ferromagnets by the spin-orbit interaction (SOI) $[4,5]$. Other proposals involve a timedependent gate [6,7], and the spin Hall effect [8].

Spin-transfer by spin-flip scattering in metal structures causes mechanical torques [9-11]. Mal'shukov et. al. [12] predict that a spin-polarized current can induce torsional vibrations in a semiconductor beam by strain-induced SOI and speculate about a possible reverse effect.

In this Letter, we propose a nano-electro-mechanical system (NEMS) that generates spins by the coupling to torsional oscillations of a freestanding semiconductor bridge, beam, or rod that is actuated, e.g., by magnetomotive [9,13], electrostatic [14] or piezoelectric [15] forces. Subject to an oscillating strain, the SOI in the semiconductor generates a spin splitting which, in the presence of a bias and impurity scattering, leads to a spin current. In analogy with piezoelectricity, in which elastic strain induces free charges, this can be called a piezospintronic effect. We illustrate the physical principle by a conducting wire in the electric quantum limit in which only a single quantized subband is occupied. Subsequently, we generalize the results to the multichannel case. We also demonstrate by numerical simulations that the effect survives the disorder expected in real systems and discuss the conditions under which it can be observed.

Let us consider a beam with length $L$ and rectangular cross section of width $d$ and thickness $a(L \gg d, a)$ (see Fig. 1) that connects two semi-infinite conducting reservoirs. Results can be easily generalized to axially symmetric rods such as catalytically grown nanowires [16]. The conducting material is a semiconductor that is grown on top of a dielectric (the semiconductor part can also be sandwiched between two identical materials of different width to eliminate the effects of structural asymmetry).
PACS numbers: 71.70.Ej, 71.70.Fk, 72.25. $-\mathrm{b}, 85.85 .+\mathrm{j}$

Without loss of generality, we assume here a structure consisting of a conducting medium on top of an insulator both being $a / 2$ thick. The leading modification of the conduction band Hamiltonian of a semiconductor due to lattice strain reads [17]

$$
\begin{aligned}
\hat{H}_{S O}= & \frac{\hbar^{2}}{2 m^{*}}\left\{\gamma \left[\hat{\sigma}_{y}\left(u_{x y} k_{x}-u_{y z} k_{z}\right)+\hat{\sigma}_{z}\left(u_{y z} k_{y}-u_{z x} k_{x}\right)\right.\right. \\
& \left.+\hat{\sigma}_{x}\left(u_{z x} k_{z}-u_{x y} k_{y}\right)\right]+\beta\left[\hat{\sigma}_{y} k_{y}\left(u_{z z}-u_{x x}\right)\right. \\
& \left.\left.+\hat{\sigma}_{z} k_{z}\left(u_{x x}-u_{y y}\right)+\hat{\sigma}_{x} k_{x}\left(u_{y y}-u_{z z}\right)\right]+ \text { H.c. }\right\},
\end{aligned}
$$

where $m^{*}$ is the effective mass, $u_{i j}$ are elements of the strain tensor, and $\hat{\sigma}_{i}$ are Pauli matrices. By focusing on narrow-gap semiconductors, we may disregard the terms proportional to the small parameter $\beta$ [17].

We are interested in the lowest energy vibrations of the beam that can be described by an isotropic elastic continuum model [18]. Elastic flexural (bending) modes cause only diagonal $u_{i i}$ strains that couple to the electrons only via the small $\beta$ term [see Eq. (1)] [12]. The strain due to torsional (twisting) vibrations is [18]

$$
u_{z y}=\tau(y, t) \frac{\partial \chi}{\partial x} ; \quad u_{x y}=-\tau(y, t) \frac{\partial \chi}{\partial z} ; \quad u_{z x}=0,
$$

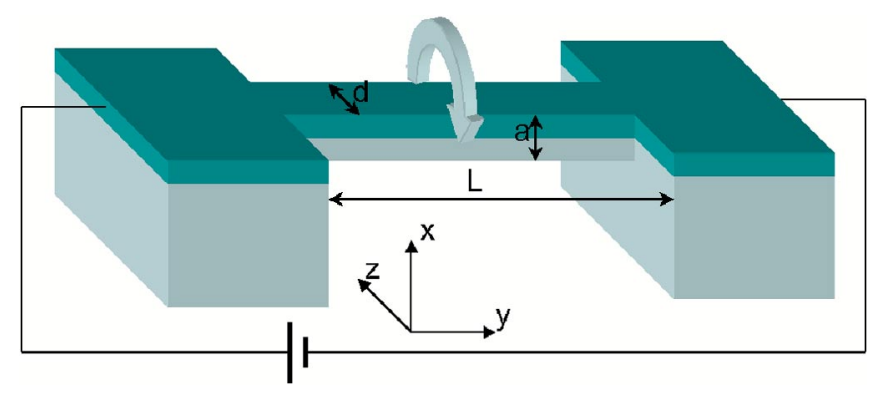

FIG. 1 (color online). A beam consisting of a semiconductor and insulator parts (semiconductor layer of thickness $a / 2$ is on top of insulator layer of the same thickness) is excited by an external source into torsional oscillations. A voltage is applied over the device to detect the mechanically induced spin splitting. 
where $\tau(y, t)=\partial \varphi / \partial y$ is the derivative of the torsion angle $\varphi$ with respect to $y$. The function $\chi$ characterizes the cross-section geometry of the beam and depends here only on $x$ and $z$. It satisfies the equation $\Delta \chi=-1$ with vanishing boundary conditions [18]. We adopt the thin plate geometry $a \ll d$ which leads to $\chi(x, z) \approx-\left(x^{2}-\right.$ $\left.a^{2} / 4\right) / 2$ and the Hamiltonian

$$
\hat{H}_{S O}=\frac{\hbar^{2}}{2 m^{*}}\left[\gamma x \tau(y, t)\left(\hat{\sigma}_{y} k_{z}-\hat{\sigma}_{z} k_{y}\right)+\text { H.c. }\right] .
$$

Equation (3) is similar to a Rashba spin-orbit Hamiltonian; however, electrons are not confined to the lowest subband in the transverse to the plane direction and the coupling strength is time and position dependent.

We now turn to the lowest electronic subband limit, disregarding intrinsic SOI, e.g., Rashba type, and assuming that the strain-induced perturbation is weak. The free-electron lowest energy states read $\Psi(x, y, z)=$ $R_{0}(x, z) \Phi(y)$, where $R_{0}(x, z) \sim \sin (\pi z / d) \sin (2 \pi x / a)$ is the lowest subband and $\Phi(y)$ is a spinor function. The projected one-dimensional Hamiltonian then reads

$$
\hat{H}_{1 D}(y)=\frac{\hbar^{2}}{2 m^{*}}\left(k_{y}-\frac{\gamma \tau(y, t) a}{4} \hat{\sigma}_{z}\right)^{2}+V(y),
$$

where $V(y)$ is the potential due to impurities and we disregarded terms $\sim(\gamma \tau)^{2}$. Electrons with up and down spins turn out to be uncoupled and subject to effective vector potentials of opposite sign, $\mathbf{A}= \pm \hbar \frac{\gamma \tau(y, t) a}{4} \mathbf{y}$. Since $\boldsymbol{\nabla} \times \mathbf{A}=0$, this vector potential does not describe an effective magnetic but a spin-dependent electric field

$$
\mathbf{E}=-\hat{\sigma}_{z} \frac{\hbar \gamma a}{4} \frac{\partial \tau}{\partial t} \mathbf{y} .
$$

The equation of motion for the torsional angle reads

$$
C \frac{\partial^{2} \varphi}{\partial y^{2}}-\rho I \frac{\partial^{2} \varphi}{\partial t^{2}}=0,
$$

where $I=\int\left(z^{2}+x^{2}\right) d z d x \simeq a d^{3} / 12$ is the moment of inertia of the cross-section about its center of mass, $\rho$ the mass density and $C$ is an elastic constant defined by the shape and material of the cantilever. $C=\frac{1}{3} \mu d a^{3}$ for a plate with $a \ll d$, and $\mu$ is the Lamé constant. The general solution of Eq. (6) is a plane wave $\varphi=\varphi_{0} e^{i \omega t \pm i k y}$, where $k=\omega / c$ is the wave number, $c=2 c_{t} a / d=\sqrt{C /(\rho I)}$, and $c_{t}=\sqrt{\mu / \rho}$ is the sound velocity. Throughout this paper, we consider a doubly clamped beam in which the lowest harmonic $\varphi=\varphi_{0} \sin (k y) \sin (\omega t)$ is excited, where $\omega=c k$ and $k=\pi / L$ is the wave number (see Fig. 1). The standing mechanical wave creates an oscillating electric field $E=\partial \mathbf{A} / \partial t$ that is exactly out of phase for spin-up and spin-down electrons. In the Born-Oppenheimer approximation, the strain induces a parametric potential $U(y)=\left(\hbar \gamma a \omega \varphi_{0} / 4\right) \sin (y \pi / L) \cos (\omega t)$ [cf. Eq. (5)] that is adiabatically followed by the electrons. The straininduced potentials $\pm U(y)$ vary only slowly and do not yet spin-polarize a charge current significantly. However, defect scattering strongly amplifies the piezospintronic effect, as illustrated now by a single short-range potential scatterer $V(y)=v \delta(y-L / 2)$ located in the middle of the beam. Disregarding the small intrinsic effect caused by $\pm U(y)$, the probability that an electron with Fermi wave number $k_{F}$ is transmitted reads [19]

$$
T_{\uparrow(\downarrow)}\left(k_{F}\right)=\frac{2\left(\hbar^{2} k_{F}^{2} / 2 m^{*} \pm U_{0} \cos (\omega t)\right)}{2\left(\hbar^{2} k_{F}^{2} / 2 m^{*} \pm U_{0} \cos (\omega t)\right)+v^{2} m^{*} / \hbar^{2}},
$$

where $U_{0}=\hbar \gamma a \omega \varphi_{0} / 4$. From the Landauer conductance formula, the spin polarization of a charge current is

$$
P=\frac{T_{\uparrow}-T_{\downarrow}}{T_{\uparrow}+T_{\downarrow}}=\frac{U_{0}\left(v^{2} m^{*} / \hbar^{2}\right) \cos (\omega t)}{E_{F}\left(2 E_{F}+v^{2} m^{*} / \hbar^{2}\right)-2 U_{0}^{2} \cos ^{2}(\omega t)},
$$

where the Fermi energy $E_{F}=\hbar^{2} k_{F}^{2} / 2 m^{*}$. The spin polarization oscillates in time with the beam frequency.

Generalization to a multichannel wire is facilitated by the local gauge transformation [20]

$$
\psi=e^{i f(x, y, t) \hat{\sigma}_{z}} \psi^{\prime}
$$

with $e^{i f(x, y, t) \hat{\sigma}_{z}}=\hat{1} \cos (f)+i \hat{\sigma}_{z} \sin (f)$, which leads to the transformed Hamiltonian:

$$
\begin{aligned}
\hat{H}= & \hbar \frac{\partial f(x, y, t)}{\partial t} \hat{\sigma}_{z}+\frac{\hbar^{2}}{2 m^{*}}\left(k_{x}+\frac{\partial f(x, y, t)}{\partial x} \hat{\sigma}_{z}\right)^{2} \\
& +\frac{\hbar^{2}}{2 m^{*}}\left(k_{y}-\gamma \tau(y, t) x \hat{\sigma}_{z}+\frac{\partial f(x, y, t)}{\partial y} \hat{\sigma}_{z}\right)^{2} \\
& +\frac{\hbar^{2}}{2 m^{*}}\left(k_{z}+\gamma \tau(y, t) x\left[-\sin (2 f) \hat{\sigma}_{y}+\cos (2 f) \hat{\sigma}_{x}\right]\right)^{2} .
\end{aligned}
$$

It is convenient to choose $\frac{\partial f(x, y, t)}{\partial y}=\gamma \tau(y, t) a / 4$. We allow many occupied subbands along the $z$ axis but restrict considerations to the lowest subband along the $x$ axis, which is the case for a laterally weakly confined twodimensional electron gas. After projecting Eq. (10) to the lowest mode in the $x$ direction, we obtain the following two-dimensional Hamiltonian

$$
\begin{aligned}
\hat{H}_{2 D}= & \frac{\hbar^{2} k_{y}^{2}}{2 m^{*}}+\frac{\hbar^{2}}{2 m^{*}}\left[k_{z}+\frac{\gamma \tau(y, t) a}{4}\left(-\sin (2 f) \hat{\sigma}_{y}\right.\right. \\
& \left.\left.+\cos (2 f) \hat{\sigma}_{x}\right)\right]^{2}+U(y) \hat{\sigma}_{z}+V(y, z),
\end{aligned}
$$

where $\quad U(y)=\hbar(\partial f(y, t) / \partial t)=\left(\hbar \omega L / l_{\mathrm{so}}\right) \sin (y \pi / L) \times$ $\cos (\omega t),(\gamma \tau(y, t) a / 4)=\left(\pi / l_{\mathrm{so}}\right) \cos (y \pi / L) \sin (\omega t), l_{\mathrm{so}}=$ $\left(4 L / \gamma \varphi_{0} a\right)$ can be interpreted as spin precession length, and $V(y, z)$ describes two-dimensional disorder scattering, $\tau(y, t)$ is here still arbitrary, but we limit our attention to the lowest vibrational mode as before.

The terms proportional to $\hat{\sigma}_{x(y)} k_{z}$ in Eq. (11) induce subband transitions; however, these do not affect transport when the precession length $l_{\mathrm{so}}$ is larger than the width of the channel. In the limit of a long and narrow beam, we may again treat the time dependence of the Hamiltonian 
Eq. (11) parametrically in terms of the frequency $\omega$. A simple short-range wall potential $V(y, z)=v \delta(y-L / 2)$ does not lead to subband transitions and we can further simplify Eq. (11) by disregarding subband transitions. Our system then reduces to a collection of independent channels, which lead to a total spin-current polarization

$$
P=\sum_{m}^{M}\left(T_{\uparrow}\left(k_{m}\right)-T_{\downarrow}\left(k_{m}\right)\right) / \sum_{m}^{M}\left(T_{\uparrow}\left(k_{m}\right)+T_{\downarrow}\left(k_{m}\right)\right),
$$

where $m$ is the channel index, $M$ is the total number of transport channels, $k_{m}$ is the wave number of an electron in the channel $m$ at the Fermi energy and $T_{\uparrow(\downarrow)}\left(k_{m}\right)$ is given by Eq. (7). In Fig. 2, we present results of Eq. (12) for the maximum mechanically induced spin-current polarization as a function of the beam width. The sharp peaks in the polarization are due to an opening of a new channel and a van Hove singularity. The dashed line in Fig. 3 are the results of Eq. (12) for the spin-polarization as a function of time.

The idealized model above allowed us to illustrate the physics of piezo-spintronics. We now consider a more realistic model, including many electron modes, subband mixing and arbitrary forms of the potential $V(y, z)$. We numerically calculate the scattering matrix using the recursive Green's function technique and the tight-binding representation of the Hamiltonian (11)

$$
\begin{aligned}
H= & \sum_{i j \sigma} \epsilon_{i j \sigma} c_{i j \sigma}^{\dagger} c_{i j \sigma}+t \sum_{i j \sigma}\left(c_{i+1 j \sigma}^{\dagger} c_{i j \sigma}+c_{i j+1 \sigma}^{\dagger} c_{i j \sigma}\right) \\
& -i t_{\mathrm{so}} \sum_{i j \sigma \sigma^{\prime}}\left(c_{i+1 j \sigma}^{\dagger} c_{i j \sigma^{\prime}}\left[-\sin (2 f) \hat{\sigma}_{y}\right]^{\sigma \sigma^{\prime}}\right. \\
& \left.+c_{i+1 j \sigma}^{\dagger} c_{i j \sigma^{\prime}}\left[\cos (2 f) \hat{\sigma}_{x}\right]^{\sigma \sigma^{\prime}}\right)+ \text { H.c. }
\end{aligned}
$$

where $\epsilon_{i j \sigma}$ is the on-site energy that includes $V$ and $U, t=$ $\hbar^{2} /\left(2 m^{*} b^{2}\right)$ is the hopping energy and $t_{\text {so }}=\hbar^{2} /\left(2 l_{\text {so }} m^{*} b\right)$ is the hopping energy due to the SOI, in terms of the tightbinding lattice spacing $b$. The bold lines in Fig. 3 display

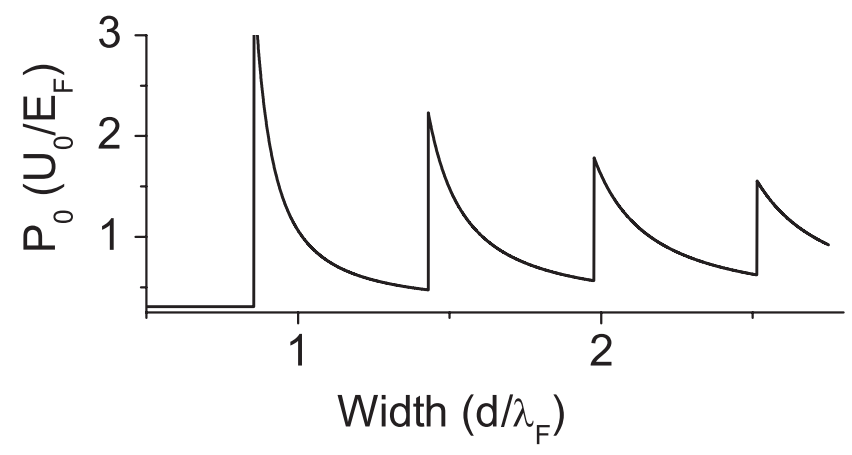

FIG. 2. Maximum spin polarization in units of the effective splitting $U_{0} / E_{F}$ as a function of the width of the single mode (along the $x$ axis) rod in Fig. 1. The frequency of the mechanical oscillations $\omega=10 \mathrm{GHz}$ which corresponds to $U_{0} / E_{F}=2 \times$ $10^{-4}$ (length of the rod $L=1 \mu \mathrm{m}$, Fermi length $\lambda_{F}=30 \mathrm{~nm}$, the impurity scattering strength $v^{2} m^{*} / \hbar^{2}=0.4 E_{F}$ and $m^{*}=$ $0.06 m)$. our numerical results for the polarization as a function of time for the short-range wall potential used above. We find good agreement with the analytical results for large aspect ratios of the beam, as expected. Unlike the spin splitting, the subband transitions are defined by the function $f(y, t)$ that is quarter of a period shifted from $U$. Therefore, the additional spin polarization due to subband transitions also has a quarter shift in Fig. 3. We also included Rashba type SOI into our numerical model and found no qualitative difference with the results in Fig. 3 when $l_{\text {so }} \ll l_{\text {Rashba }}$ where $l_{\text {Rashba }}$ is the precession length due to the Rashba SOI.

Finally, we model the potential $V(y, z)$ by on-site Anderson disorder with energies distributed over a band width $W$. The beam is represented as a $150 \times 9$ discrete lattice and an ensemble averaging over 20000 impurity configurations is carried out. A single realization (without averaging) behaves similar to our single defect result in Fig. 3. Averaged results are presented in Fig. 4. The Anderson disorder strength can be measured by an effective 2D mean free path [21] as $l_{2 \mathrm{D}}=\left(6 \lambda_{F}^{3} E_{F}^{2}\right) /\left(\pi^{3} a^{2} W^{2}\right)$. Here we consider $l_{2 \mathrm{D}}=8 L, 3.6 \mathrm{~L}$ and $2 L$ (disorder scattering in 2DEGs is usually caused by surface roughness and ionized donor impurities which can be controlled by crystal growth techniques and sample design, e.g., remote doping). When the Anderson disorder is weak, the spin polarization is almost a harmonic function of time. We may conclude that the piezospintronic effect is very robust, persisting in a disordered system, confirming the qualitative behavior of the analytical model with one dominating defect scatterer. In fact, contrary to the earlier proposals $[6,7]$, our device can benefit from moderate disorder.

The choice of parameters above is motivated by the following estimates made for a silicon cantilever of size

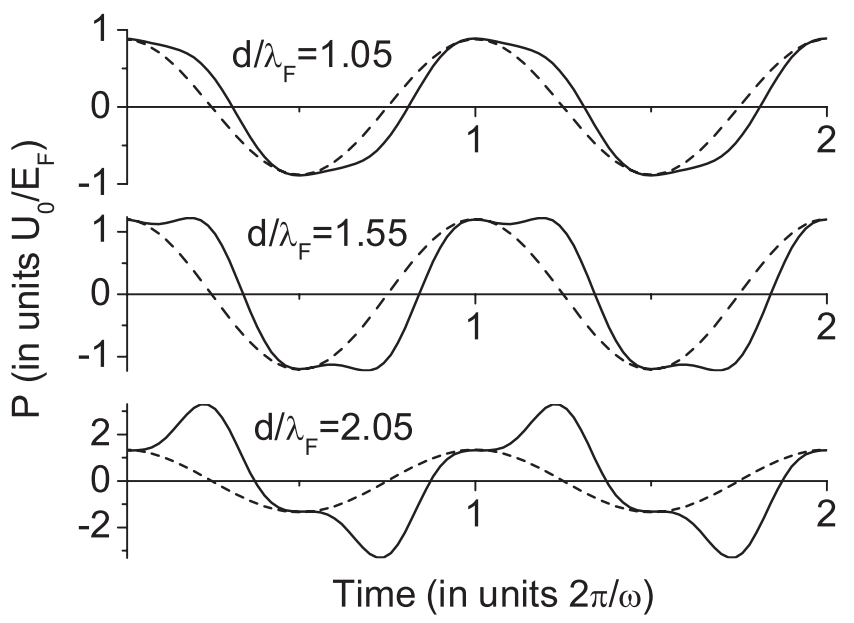

FIG. 3. Spin-current polarization in units of the splitting parameter $U_{0} / E_{F}$ as a function of time for the torsional oscillations of the single mode (along the $x$ axis) rod in Fig. 1. The dashed line gives the results according to Eq. (12), whereas the bold line represent the results of numerical simulations based on the tight binding model. Parameters are the same as in Fig. 2. 


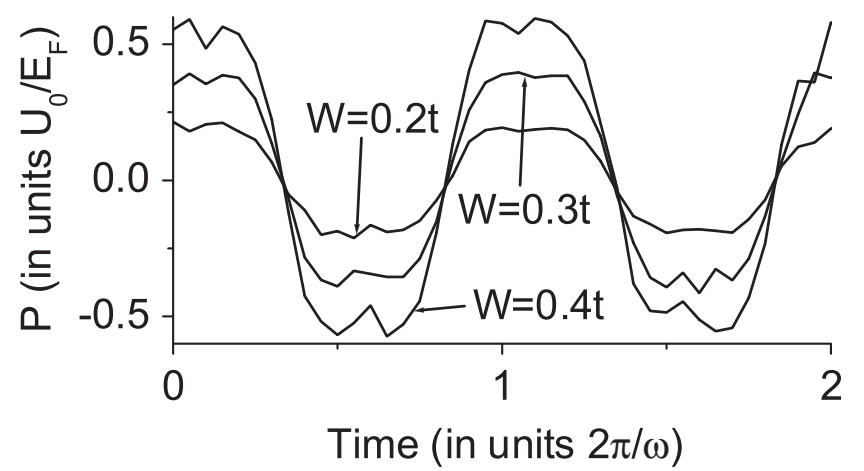

FIG. 4. Spin-current polarization in units of the effective splitting $U_{0} / E_{F}$ as a function of time for the torsional oscillations of the $2 \mathrm{D}$ beam with Anderson disorder. Parameters are the same as in the other figures.

$a \times d \times L=(0.05 \times 0.15 \times 1) \mu \mathrm{m}$. The resonant frequency is $\omega=c \pi / L=10 \mathrm{GHz}$, using the density of silicon $\rho=2 \times 10^{3} \mathrm{~kg} \mathrm{~m}^{-3}$ and the Lamé constant $\mu=$ $100 \mathrm{GPa}$. The maximum angle of torsion $\varphi_{0}$ can be estimated by equating the energy dissipation during a cycle $2 \pi \varphi_{0}^{2} C /(Q L)$, where $Q$ is the mechanical quality factor, with the energy input rate $\varphi_{0} T$, where $T$ is the actuating torque applied at the center of the beam. Electrostatic torques of $T \sim 10^{-12}-10^{-15} \mathrm{~N} \mathrm{~m}$ have been already realized [14]. Taking $Q=500, T=10^{-15} \mathrm{Nm}$ and $C=$ $10^{-18} \mathrm{~N} \mathrm{~m}^{2}$, we find $\varphi_{0}=0.2 \mathrm{rad}$. The corresponding spin-orbit precession length is $l_{\mathrm{so}}=1 \mu \mathrm{m}$ (which is comparable to a typical semiconductor 2DEG) and the spin splitting is $U_{0}=\hbar \omega L / l_{\text {so }} \approx 6 \times 10^{-6} \mathrm{eV}$, using the bulk strain-SOI parameter for GaAs $\gamma=2 \times 10^{8} \mathrm{~m}^{-1}$ [17] which is not expected to be affected strongly by quantum confinement [22]. This leads to polarizations of the order of $P \sim 10^{-4}$. Larger polarizations $P \sim 10^{-3}$ and spin splittings $U_{0} \approx 6 \times 10^{-5} \mathrm{eV}$ are expected in $\mathrm{InSb}$ with the narrower semiconductor gap $E_{g}$ and larger spin-orbit splitting $\Delta\left(\gamma \sim \frac{\Delta}{E_{g}}\right)$. Note that the finite temperature comparable to the spin splitting should weaken the effect. In the presence of an applied dc voltage, the mechanical motion generates an ac spin current. Alternatively, one can apply an ac voltage synchronized with the mechanical motion to obtain a dc spin current. This ac voltage can be a result of piezoelectric effect allowing pumping of spins by a mechanical oscillation in unbiased structures. The created spin accumulation can be detected by ferromagnetic contacts [23] or optical Kerr rotation [24].

By tuning the doping density and the width of the beam, it is possible to take advantage of the resonant structure in Fig. 2 to increase the spin signal. Rods with higher quality factors can have larger amplitude of oscillations leading to higher polarizations.

Summarizing, we propose a piezospintronic effect that is based on strain-induced coupling of the electron spin degree of freedom and mechanical vibrations in freestanding semiconductor nanobeams. We show that time-dependent strain due to torsional mechanical oscillations can lead to a measurable spin polarization of an applied charge current. Mechanically generated spin-dependent potentials (mechanically induced Zeeman splittings) can be also used for the manipulation of an applied spin currents. We propose ways to measure and increase such mechanically generated polarization that can be used for effective spin injection in spintronic based devices.

We thank Ar. Abanov, K. Výborný, and T. Jungwirth for helpful discussions. This work was supported by ONR under Grant No. 000140610122, by NSF under Grant No. DMR-0547875, and by SWAN-NRI. J. S. acknowledges support from the Cottrell Scholar Program of Research Corporation.

[1] I. Žutić, J. Fabian, and S. Das Sarma, Rev. Mod. Phys. 76, 323 (2004).

[2] A. Brataas et al., Phys. Rev. B 66, 060404 (2002).

[3] Y. Tserkovnyak et al., Rev. Mod. Phys. 77, 1375 (2005).

[4] V. M. Edelstein, Solid State Commun. 73, 233 (1990).

[5] Jun-ichiro Inoue, G. E. W Bauer, and L. W. Molenkamp, Phys. Rev. B 67, 033104 (2003).

[6] M. Governale, F. Taddei, and R. Fazio, Phys. Rev. B 68, 155324 (2003).

[7] C.S. Tang, A. G. Mal'Shukov, and K.A. Chao, Phys. Rev. B 71, 195314 (2005).

[8] S. Murakami, N. Nagaosa, and S.-C. Zhang, Science 301, 1348 (2003); J. Sinova et al., Phys. Rev. Lett. 92, 126603 (2004); Y. Tserkovnyak et al., Phys. Rev. B 76, 085319 (2007); I. Adagideli and G. E. W. Bauer, Phys. Rev. Lett. 95, 256602 (2005); T. Kimura et al., Phys. Rev. Lett. 98, 156601 (2007).

[9] P. Mohanty et al., Phys. Rev. B 70, 195301 (2004).

[10] P. Fulde and S. Kettemann, Ann. Phys. (Leipzig) 7, 214 (1998).

[11] A. A. Kovalev, G.E.W. Bauer, and A. Brataas, Jpn. J. Appl. Phys. 45, 3878 (2006); A. A. Kovalev, G.E.W. Bauer, and A. Brataas, Phys. Rev. B 75, 014430 (2007).

[12] A. G. Mal'shukov et al., Phys. Rev. Lett. 95, 107203 (2005).

[13] X. M. H. Huang et al., New J. Phys. 7, 247 (2005).

[14] A. M. Fennimore et al., Nature (London) 424, 408 (2003).

[15] S. C. Masmanidis et al., Science 317, 780 (2007).

[16] X. F. Duan et al., Nature (London) 409, 66 (2001).

[17] G.E. Pikus and A.N. Titkov, in Optical Orientation (North-Holland, Amsterdam, 1984).

[18] L. Landau and E. Lifshitz, Theory of Elasticity (Pergamon, New York, 1986), 3rd ed., Vol. 7.

[19] L. Landau and E. Lifshitz, Quantum Mechanics (Butterworth-Heinemann, Oxford Boston, 1991), 3rd ed., Vol. 3.

[20] G. Tatara, H. Fukuyama, Phys. Rev. Lett. 78, 3773 (1997).

[21] T. Ando, Phys. Rev. B 44, 8017 (1991).

[22] R. Winkler, Spin-orbit Coupling Effects in 2-dimensional Electron and Hole Systems (Springer, Berlin, 2003).

[23] X. Lou et al., Phys. Rev. Lett. 96, 176603 (2006).

[24] Y. K. Kato, R. C. Myers, A.C. Gossard, and D.D. Awschalom, Science 306, 1910 (2004). 